\title{
Designing Sharing Economy Platforms through a 'Solidarity HCl' lens
}

\author{
VASILIS NTOUROS, Open Lab, School of Computing, Newcastle University, UK \\ HARA KOUKI, Department of Sociology, School of Social Sciences, University of Crete, Greece \\ VASILIS VLACHOKYRIAKOS, Open Lab, School of Computing, Newcastle University, UK
}

\begin{abstract}
Despite sharing economy's promise of a novel, inclusive and community building socio-techno-economic system, sharing economy has been indicted, among others for profiteering from previously private and occasionally non-monetized activities, for turning the activity of sharing into an individualistic and impersonal one, for reproducing stereotypes and creating precarious jobs. In the epicenter of such critiques are the 'big' and 'limelight-ed' platform-firms, like AirBnB and Uber and the digital infrastructures they employ. To the best of our knowledge, the majority of related research focuses on SE platforms of this ilk. In response, acknowledging that the problem is not the agency of the digital in the activity of sharing per se, but that the wrong people set the terms, design and benefit from this mediation, we find it timely to explore the existence of community-driven sharing economy initiatives and explore how they use the digital to support their sharing ends. As a result, in this paper we report from our engagement with a ride-sharing initiative, called 'Share the ride ;)' which operates within a Facebook group since 2009 and is the most popular ride-sharing 'platform' in Greece. Extrapolating from our findings and while adopting a 'Solidarity HCI' approach, a call to design for 'human' rather than market needs, we participate in the 'sharing discourse' by providing design implications for the development of sharing economy platforms which can favor community building, participation, self-organization and the nurturing of a generative sharing ideology. To this end, we suggest the development of malleable sharing economy platforms and of mechanisms which can support the development of relational trust(s) and enduring social relations. Finally, we underscore that in order to favor the establishment of such relations, those platforms should employ architectures which esteem pluralism and self-affirmation.
\end{abstract}

CCS Concepts: • Human-centered computing $\rightarrow$ Collaborative and social computing systems and tools.

Additional Key Words and Phrases: sharing economy, ride-sharing, solidarity HCI, malleability, relational trust, self-affirmation

ACM Reference Format:

Vasilis Ntouros, Hara Kouki, and Vasilis Vlachokyriakos. 2021. Designing Sharing Economy Platforms through a 'Solidarity HCI’ lens. 1, 1 (January 2021), 25 pages.

\section{INTRODUCTION}

Except those described as infinite resources (e.g. the air we breathe, the sun) which are shared among 'humans' and all other earthlings, sharing of finite resources has been described as an a priori condition for "humans" coexistence, the foundation of community organization [4] and a routine activity of which values every child learns at an early stage of their life [75]. M. Felson and Joe L.Spaeth in 1978 [27] referred to the sharing of such resources by the term

Authors' addresses: Vasilis Ntouros, v.ntouros2@newcastle.ac.uk, Open Lab, School of Computing, Newcastle University, Urban Sciences Building, 1 Science Square, NE4 5TG, Newcastle upon Tyne, UK; Hara Kouki, kouki@uoc.gr, Department of Sociology, School of Social Sciences, University of Crete, University Campus, 74100, Rethymno, Greece; Vasilis Vlachokyriakos, Open Lab, School of Computing, Newcastle University, Urban Sciences Building, 1 Science Square, NE4 5TG, Newcastle upon Tyne, UK.

Permission to make digital or hard copies of all or part of this work for personal or classroom use is granted without fee provided that copies are not made or distributed for profit or commercial advantage and that copies bear this notice and the full citation on the first page. Copyrights for components of this work owned by others than ACM must be honored. Abstracting with credit is permitted. To copy otherwise, or republish, to post on servers or to redistribute to lists, requires prior specific permission and/or a fee. Request permissions from permissions@acm.org.

(1) 2021 Association for Computing Machinery.

Manuscript submitted to ACM 
'collaborative consumption' which they used to describe events during which people consume finite goods through joint activities.

Y. Benkler describes two characteristics of goods and resources which determine their shareability [11]. They are shareable if (i) lumpy: they are purchased as is -thus are indivisible- and they are more powerful than the need they were obtained for (e.g. the power of computers that have processors and storage disks beyond what most users will require [82] and if (ii) they possess medium granularity: are small enough for an individual to justify buying for their own use. An example of such mid-grained goods which as also lumpy cannot be used to their full capacity are private cars that are not driven 24 hours a day and of which seats are not used constantly. If goods/resources have those two attributes, they can be considered underused, thus 'shareable'. This circumstance, 'shareability', opens up new possibilities for their management.

Within Web 2.0 which has changed the ways people connect to each other and share information, those management possibilities have been considerably enhanced. In this light, information exchange about the above-described as 'shareable', brings us closer to what today is marked as the sharing economy (SE). In SE, digital systems, usually called SE platforms (SEPs) or peer-to-peer exchange platforms/markets, are developed to mediate the exchange of all kinds of resources. SEPs range from for-profit and transactional platforms which support markets like Airbnb and Uber within which sharing coincides with renting and hiring, to non-transactional platforms like Couchsurfing, to less statutory and informal, occasionally more self-organized SE schemes which operate on top of existing ICTs (e.g. on social media). Due to this breadth of SEPs and because of the "semantic confusion" [10] around the term 'sharing', L. Richardson [72] describes the SE as a "paradox" that encompasses both a capitalist, business as usual, economy and its alternatives; a space for 'constructing' opportunity while 'deconstructing' the dominant capitalocentric practices.

Researchers in HCI and elsewhere, have shown extended interest in the SE; inter alia in discussing what can be put under the SE umbrella and what should not [32,76], in offering policy proposals[50, 71], in exploring how the SE is affecting architecture and urban planning [20], in discussing SE's impact on mobility and transportation [12, 42, 85] housing [8, 44, 47], labor [21, 75, 77], social relations [22, 32, 39, 66]. Despite this gamut of SE related works, to the best of our knowledge, the majority of research centres on the 'big' and 'limelight-ed' SEPs which with very few exceptions have been highly criticized as 'sharewashing', extractive and exploitative neoliberal projects [18, 75, 76] -embodied in digital designs- which create precarious jobs [75, 78] reproduce inequalities and discrimination [17, 26, 86], spread market relations to our lives, monitor and profiteer from previously private and occasionally non-monetized activities [75].

While acknowledging that similarly to all types of markets and other infrastructures, SEPs do not arise as natural phenomena but are deliberately designed "social product[s]" [24,73] materialized in certain technologies, we believe that there is a lack of understanding on how the digital is being used by SE initiatives to support the sharing of resources (outside the framework of SE as described above) as a community-driven, caring-based, [52, 53], participatory and more democratic [75] activity. In this regard, responding to P. Allen's design-centred call for further research on SE that explore "what difference does it make if mediation takes one form vs. another?" [1], in this paper we report on our work with a community-driven ride-sharing initiative which operates within a Facebook (FB) group called "Share the ride ;)" (StR). StR, despite the fact that -similarly to all FB groups- has not been designed to mediate sharing of physical resources per se, has evolved into a ride-sharing 'platform' that is designed and managed as such, internally by its members.

To explore how the activity of ride-sharing is performed within StR's sociotechical SE environment, we took as a starting point A.Sundarajan's [82] understanding of SEPs, as a set of technologies and practices (the majority of Manuscript submitted to ACM 
which are enforced by these technologies) that enable the sharing of 'latent' resources through 'facilitating' their search and discovery, organizing the logistics and the creation of trust. In specific, we focused on how those phases manifest among StR members and which sociotechnical designs support them. Accordingly, we have been interested to explore how StR emerged in FB and how it has evolved into a community-driven SE 'platform'.

In order to explore the abovementioned, we undertook a series of semi-structured interviews from members of StR which hold different roles in the group.

We found (as we present in our findings and discussion sections), that StR members have been harnessing FB's flexible (in the context of ride-sharing) architecture to develop sociotechnical processes in order to better support ride-sharing as a service and to better support the group's inclusive and participatory character. Moreover, we found that StR members build relations and trust(s) in plural ways due to the negotiations that manifest both among individuals as riders when setting up the logistics of a ride-share and among StR users as members of a broader community.

Departing from those insights which come in contrast to the relatively fixed, impersonal and non-participatory ways that SE is performed in mainstream SEPs [52,75, 76], in our discussion section, while adopting a 'Solidarity HCI' approach -a call to design for 'human' rather than market needs [87], we contribute design implications for the development of SEPs as infrastructures which can favor the incubation of a generative sharing culture and which can become the basis for the emergence of multiple SE schemes and initiatives. Initiatives that can be more self-organized as to what can be shared, to the digital designs used to support the sharing activity, to building trust(s) and identities. To this end, we propose malleability as a design quality, the development of tools and processes that can favor relational trust evidenced in repeated interactions among sharers and the employment of designs that can foster pluralism and self-affirmation.

\section{RELATED WORK}

The 21st century SE, broadly refers to an economy based on the use of digital platforms via which users can exchange all kinds of resources and assets that are considered to be idle. Such can be one's tools (e.g. a driller), a few empty seats of one's vehicle and also one's spare time which can be made available via those platforms; it can be offered without a monetary compensation to help a stranger, say, to learn how to play the violin or it can be sold as labour while driving around strangers in a city with a private car.

Thus, under the "big tent" of SE [76] one may find various technological systems -with varying affordances- which underpin different SE business models. As those different SE models are embodied by different value-driven technologies, their architectures play a central role [51]. For example, Airbnb is supported by a digital system that serves the platform's for-profit needs and supports the commercialization of sharing while Couchsurfing -for-profit but non-transactionalemploys another system that also differs from the one used by the platform Freecycle.org.

Except for a digital system that holds the potential to create a new decentralized market [82] and the 'idling' capacity of assets and resources, according to R. Botsman and R. Rogers [14] a SEP in order to function needs a critical mass of users who despite being strangers can trust each other. Extending this, Lampinen and Brown [51] use the term 'thickness' to note that SEPs do not only need a critical mass of participants but also the 'right' kind of participants who can find among each other suitable exchange partners.

Along critical mass and 'thickness', search and discovery, trusting each other and organizing the logistics are the three core and interrelated phases which A.Sundarajan identifies as central to a SEP's operation [82]. Search and discovery refers to the process a SEP user has to go through in order to find the available/ 'shareable' resources that they are interested in (e.g. a room, a tool, a seat in a vehicle). The majority of SEPs offer their users the ability to 'upload' 
information about the resources they want to share; either resources they own or want to have access to. Trusting each other refers to the phase during which the parties (the one that offers and the one in need) have to trust each other in order to agree on participating in a sharing activity. Within this phase, reviewing and rating systems are usually employed. Those systems can work as traces of the past activity of a user within a SEP and are employed in order to mitigate possible risks within a strangers' sharing. As a last phase, when, say, two peers decide that they can 'trust' each other and have agreed on a sharing activity, they have to organize the logistics of this activity. For example, if two parties have agreed to participate in a ride-share they also have to agree on things like a meetup location, the possible cost of the trip, the route etc. In most SEPs when a 'sharing' activity has occurred, the participating parties have the option to review each other on the SEP. If so, those reviews will become available to future sharers. Further to the above-mentioned, how those phases are digitally supported [48] (e.g. what users can 'upload' as information about themselves and their shareable assets and in which form, what mechanisms are used to build trust(s), via which mechanisms can users communicate among each other and the platform managers) affects how the sharing activity is mediated and performed.

\section{$2.1 \quad \mathrm{HCI}$ and the SE}

2.1.1 SE's lost promise. One of the most publicized hallmarks of the SE has been its capacity to support community building, to favor inclusion and access -as ownership of assets is reconsidered [13] - and to contribute towards the mitigation of the nowadays' environmental crisis [14, 76, 82]. However, this promising narrative has been proven problematic, as studies have shown that major SE companies have failed to enact social tying [28], while also widespread access to occasionally cheaper services can increase mobility and accordingly emissions [76]. For example, a large-scale measurement study [47] found that most listings on Airbnb among many countries are entire homes and depicts Airbnb as a rental marketplace rather than a spare-room sharing platform that can foster community building. In the same vein, a 2017 EU report on the SE concludes that "sharing rarely fulfills the promise of generating social capital" and that "sharing in Europe is, above all, a business" [2].

Being critical towards SE $[79,82]$, suggest that within this modality, the lines between the personal and the professional are blurring and new forms of precarious labour are emerging. Cockayne [18] while focusing on those new forms of labour mediated by for-profit and transactional SEPs within which the 'shared' is one's labour (e.g. Uber, Lyft, TaskRabbit) underscores that those, of neoliberal ilk platforms promote labour as something that is 'shared', not sold and purchased. Thus, labour becomes "metonymic" [18] with cheap, always-available information. T. Sholz describes big for-profit SE as an on-demand service economy that "is spreading market relations deeper into our lives", that monetizes -through secondary markets- previously unmonetized interactions (e.g. hosting a friend, giving a ride to someone) from which it also "sucks value" and which by virtue of the systems in use, keeps track (in the form of digital data) of those previously private interactions[75].

According to P. Romer because of this type of a 'sharewashing' economy[45], in which sharing is exploited due to its affectionality and positive symbolic meaning, we may be losing a good verb [76]. However, J.Schor in a less annihilating tone, remarks the potential for truly decentralized forms of "genuine sharing" and cooperation stating the need for democratizing the ownership and governance of those platforms [76, 77].

2.1.2 'Reclaiming' the SE. A.Lambinen and B.Brown [51] underscore the centrality of the digital in today's peer-to-peer exchanging platforms and thus they call HCI researchers to have an active role in designing and intervening when such platforms are not effective or do not operate on a fair basis.

Manuscript submitted to ACM 
M. Heidaripour and L. Forlano [56] based on accounts which indicate that SEPs are oftentimes contributing to the reproduction of structural inequalities with regards to gender, race, class $[9,26,74]$ argue that Feminist Science and Technology theories, practices and critics can contribute towards the development of a more inclusive and equity-based SE.

Additionally, G.Avram et al [5], but for their strong criticism against mainstream SE's impersonal and exploitative nature, suggest $\mathrm{HCI}$ researchers who are interested in reshaping SE "to think beyond the current dominant narratives of sharing and move towards care as a notion and a social practice".

Care is also central to A. Light's accounts [52,53] which accordingly underscore that big for-profit SE networks, as highly technologized, occasionally sideline the social aspect of sharing by removing "the personal element out of negotiations" and by limiting physical interaction among sharers [52]. In response, departing from their neighbourhoodscaled research, A. Light argues in favour of an 'infrastructuring' of local SE [53]; a situated social and technical process that meshes the digital with the physical and holds the potential to promote a generative culture of cooperation and sharing as a caring-based practice. Overall, A. Light argues that within digitally mediated sharing, technology should be used to help, not to replace interpersonal face-to-face activities and negotiating trust with others[52].

In a similar vein, Vlachokyriakos et al. [87] based on their research within solidarity movements discuss the implications of transferring the observed solidarity practices and values in HCI. Their research findings, suggest that solidarity movements are situated communities that build economies within which self-organization, agonism, pluralism, collaboration and sharing practices are central. In accordance with A. Light's accounts that describe a generative culture of cooperation within caring-based SE, a fundamental part of solidarity movements and economies is their "pedagogic and political awakening character" [87].

Solidarity is accordingly central to T.Scholz's concept of Platform Cooperativism [75] who recognizes that the problem is not the agency of the digital in the activity of sharing per se but that the wrong people design, set the terms and benefit from this mediation. In this regard, the concept of Platform Cooperativism offers design proposals that aim at changing ownership of SEPs, establishing democratic governance and revitalizing solidarity. Among others, within Platform Cooperativism, Scholz proposes a collective type of ownership of SEPs, argues in favour of transparent data (e.g. how user's data are treated), underscores that SEPs' users should be able to directly communicate among each other and be involved in the design process of the platform; one that rejects constant surveillance which currently occurs via data storing mechanisms and review/rating systems.

Sholz, alike [52, 76, 87, 88], conceives of SEPs as socio-technical infrastructures of which architectures are informed by political decisions and calls the designers to understand the sociology and anthropology of their creations and the possible ramifications that those designs might have.

While embracing that call, as HCI researchers who acknowledge SE's lost promise and who stand with those who want to "reclaim" the SE, in this paper we built upon those accounts and we participate in the 'sharing discourse' by presenting our findings from engaging with a community-driven ride-sharing scheme and by providing design implications for the development of SEPs through a 'Solidarity HCI' lens.

\subsection{Ride-sharing}

Ride-sharing, also known as carpooling includes hitchhiking and refers to the joint travel of two or more people in a single vehicle [34]. Ride-sharing is considered a practice "as old as the car itself" [84] which proliferated during World War II and the 1970's energy crisis [29] and during the Civil Rights Movement of the 1950s in the racially segregated communities of USA's south [31]. It can also be found in the form of the 'employer vanpool programs' [65] and as 
'slugging' in the USA [16]. Ride-sharing has historically been a non-profit driven practice, performed in a self-regulated fashion which refers to the sharing of the space in a vehicle and usually to the equal (per occupant) split-up of the total involved costs (i.e. fuel, tolls, parking costs) [11].

Nowadays, ride-sharing is one of the basic activities that is mediated by digital platforms. Agile or real-time ridesharing is supported by platforms like Uber and Lyft, while long-distance ride-sharing, by platforms like BlaBlacar Ride-sharing schemes, except when supported by bespoke systems (e.g. those used by Uber, Lyft,Blablacar), they oftentimes operate on top of various ICTs -from phone calls to social media networks; tools which are not designed to support ride-sharing per se [83]. For example, Telegram service, due to the fact that its users do not need to link their accounts to a phone number, has been recently appropriated for 'safe and emergency carpooling' during the Hong Kong protests [36]. Similarly, on FB, one can find dozens of groups used to mediate ride-sharing, both long-distance and real-time. Among others, the FB group Arcade City ${ }^{1}$ that operates in Austin (USA), the FB group Tyson's Lifts4Friends ${ }^{2}$ which operates in Canberra (Australia), and a similar FB group used in Bournemouth, Dorset (UK) [58]. Those three groups use FB to mediate real-time ride-sharing and bear a resemblance to Uber or Lyft as its members participate in on-demand short distance taxi-like services. Accordingly, there also exist groups which harness FB networking possibilities to mediate long-distance ride-sharing like Skjutsgruppen ${ }^{3}$ that operates in Sweden and StR ${ }^{4}$; the group we engaged with during this research.

2.2.1 Context: Ride-sharing in Greece. According to a 2017 report on consumer issues in peer-to-peer platform markets [40], in Greece two digital platforms ${ }^{5}$ used to mediate ride-sharing were identified; supported by the then Ministry of Environment, Energy and Climate Change through its "Sustainable Mobility Aims"[63]. None of these two platforms are functional at this time and other ride-sharing platforms that have been used in the recent past are also not accessible ${ }^{6}$. Moreover, Blablacar or similar ride-sharing firms do not operate in Greece and Uber suspended its services in April of 2018 after the ratification of the legislation $4093 / 2012^{7}$.

We also find it relevant to point out that since 2008 Greece has gone through a long socioeconomic crisis. This crisis affected the transportation and mobility sector accordingly [59]. The use of private vehicles declined [60,61, 81] while the public transport's funding, maintenance and quality of services were seriously affected [67] despite a recorded shift to the use of public transportation and of other more economic, environmentally friendly and socially alternative modes [35]. During those years and as a response to the austerity measures, new solidarity initiatives have been formed (e.g. time banks, no-middlemen goods distribution networks, soup kitchens, work cooperatives, social clinics and pharmacies, solidarity schools, alternative currencies, self-managed factories and artist collectives) [3, 70, 87]. Similarly, transport-related mobilisations and movements also emerged. For example, an informal ticket sharing movement among the users of public means of transportation, and ride-sharing initiatives like StR which was created in 2009. StR is to this moment, the most popular ride-sharing scheme in Greece ${ }^{8}$. We do not argue that this context per se incited

\footnotetext{
${ }^{1}$ Arcade City Austin Fb group can be accessed in this link: https://www.facebook.com/groups/ArcadeCityAustin/

${ }^{2}$ Tyson's Lifts4Friends Fb group can be accessed in this link: https://www.facebook.com/groups/1438415459765446/

${ }^{3}$ Skjutsgruppen FB group can be accessed in this link: https://www.facebook.com/skjutsgruppen/?tn-str $=\mathrm{k}^{*} \mathrm{~F}$

${ }^{4}$ StR FB group is accessible in this link: https://www.facebook.com/groups/sharetheride/

${ }^{5}$ carpooling.gr and hopinside.com

${ }^{6}$ i.e. pamemazi.gr, carshare.gr

${ }^{7}$ According to $4093 / 2012$ a private car and a driver can be leased for a duration not less than 6 hours and the two parties (driver and passengers) have to sign a contract in which the terms of the agreement are explicitly demonstrated

${ }^{8}$ Except for StR there also exist other FB ride-sharing groups which are used to cover the transportation needs of people travelling in geographically specific areas e.g. Share the ride Patras, Share the ride Crete, Volos Carpooling etc

Manuscript submitted to ACM
} 
StR to appear, however we find it relevant to place, present and accordingly research StR within the above described genealogy.

2.2.2 Share the ride ;) (StR). StR is a FB group which was created in October 2009 in order to facilitate long-distance ride-sharing mostly in mainland Greece. StR is the most popular ride-sharing scheme in Greece and has approximately 39.500 members (by March 2020). From August 2018 until August 2019, members posted 17.143 times and commented 30.988 with around 100.000 reactions (likes etc) ${ }^{9}$.

StR originated due to its creator's personal need to share rides. According to them

"[...] I have been trying through my own FB profile to find friends from FB to share a route, a specific route I' ve been doing a lot back in 2009. Then I thought, why shouldn't I expand somehow this attempt by making a group where friends can add their friends and possibly be able to find co-riders. [...] this is how StR was created. Bit by bit with friends and friends of friends [...] there was no advertising or anything similar. Only via world of mouth. I named the group Share the Ride;), as carpooling is not a popular term among Greeks. Then I also decided to put this emoticon, the winking one, to make it more cute and maybe less emphatic"10

Since its very beginning in 2009 and until 2015 the group had only one admin, its creator. One more admin was later added in 2015 who was a personal friend of the creator. This second admin was added while StR's creator "[...] wanted to clock out from FB for a while, so someone should keep the group alive".

Since 2019, the admin team is growing bigger and more StR members have been added due to the upsurge in members and the increased needs for the group's daily operation.

StR was initially created as an open group (non-members could access the group and the circulating content) but in July 2019, the admin team made it a closed one; only members can see the shared content.

The group is described as one for people willing to co-travel by sharing a vehicle and the related expenses. The rules (visible on the group's about page) are: "Kindness"; "No hate speech/bullying"; "No promotions or spam"; "Diligence (when setting up a ride)"; "Relevance of the posts"; "Uniformity of the posts: Accepted would only be the posts that use the \#hashtags system."

By this last rule that was introduced by the admin team in Spring 2019, StR members when doing a ride-related post are asked to use either "\#looking-for" or "\#offering" depending on their status and hashtag (\#) the destinations they are starting, heading to, intent to stop in order to leave or pick co-riders and to accordingly hashtag (\#) the date they plan to travel. The rest of the post can have the format (i.e text, photo, video) and the content that the person doing the post decides.

A typical post offering a ride-share on StR looks like the following

\#offering \#Thessaloniki \#Larissa \#Farsala \#Friday11102019 afternoon-ish. 1-2 seats available depending on the amount of luggage (FB post made by Maria Theokopoulou, 22/09/2019) ${ }^{11}$

Via this system, StR members are prompted to utilize the search button to 'filter' their search each time they are looking for co-riders.

The admin team has also proposed the use of the hashtags system for 'reviewing' members, however as of the time of writing, this method has only been used by very few members of the group.

\footnotetext{
${ }^{9}$ Those figures were provided by the admin team of the group, and were collected via the 'group insights' FB tool

${ }^{10}$ Those quotes derive from the interview we took during this research from the creator of the group to who we refer to as P3 in our Findings section

${ }^{11}$ The names of the cities/towns and the name of the person that did the post are aliases
} 


\section{METHODOLOGY}

The lead author of this paper has a longitudinal affiliation with StR prior to the undertaking of research within it. They have been a member of StR since 2015 and have participated (by December 2020) in more than 20 rides organized via the group, both as a driver and a passenger. As all StR members are FB users, the lead author participates in the group with their FB identity which in their case is a pseudonym.

The first author's previous experience with the group, played a significant role in our motivation to work with StR (as opposed to other similar groups), in the formation of an interview guide which was used to perform semi-structured interviewees and in the analysis of the collected data.

Our method of engaging with the group and data collection entailed the following stages:

(i) In early 2019, the lead author made a post on the group's wall in order to publicly communicate to StR members their research intentions. This post was made via the first author's FB profile, while also citing the 'real' (rather than the FB pseudonym) name of the researcher. This post raised 395 reactions (either thumbs up or hearts) and 122 comments, the majority of which by StR members expressing their willingness to contribute to this research.

(ii) Few days later, the first author, via another FB post, shared on the group's wall an anonymous questionnaire for members to complete. This questionnaire was used in order to collect socio-demographic data (e.g. age, gender, location, occupation, tenure in StR, affiliation with the group), gain insights from StR members about the group's use and operation and eventually recruit members for further data collection via interviews. 172 StR members completed the questionnaire out of which 42 stated their availability to be interviewed. From them, 35 participants provided adequate contact information while the rest either provided wrong contact details or no details at all.

These 35 comprised of: 10 members-passengers; 11 members-drivers; 3 members that use StR as both passengers and drivers; 11 members of StR who have not been in a ride yet.

As in the questionnaire there was not an option for participants to indicate their identity as members of the admin team nor (due to the participants anonymity) could we have found out if any of the admin team's members have filled in this questionnaire, we also contacted in person (via FB) all admin team members at that time; their FB profiles are displayed in the about page of the group. From them, four persons agreed to participate in our research.

iii) We then started taking semi-structured interviews. Conducting semi-structured interviews was chosen as a method of collecting data in order to support open-ended and reciprocal discussions between the interviewer and an heterogeneous sample of participants [46].

In relation to the order in which the interviews would be held, we chose to mix people with different affiliations and roles in the group (i.e. members of the admin team, drivers, non-drivers, members that have not been in a ride yet) in order to obtain a diverse perspective of the group's operation early on in the interviewing process.

After we have interviewed 3 drivers, 3 passengers, 2 members of the admin team and 2 members who have not yet been in a ride organized via StR we decided to stop collecting more data as we reached saturation (i.e. we kept hearing similar answers and comments) and as we have already gained insights into the sociotechnical processes that manifest within StR which were not evident to us at the beginning of this work and which within our research framework and aims could contribute to interesting implications for design to CSCW and SE research in general.

For the semi-structured interviews we created an interview guide that was used to support and direct conversations with our participants towards the different activities and processes that manifest within StR which we have been interested in exploring. The interview guide was also designed as a participant-oriented one, as we engaged with an heterogeneous sample of StR members which have different roles and experiences in the group. 
As one of our aims was to explore how different aspects of ride-sharing in particular are performed via StR, part of the guide has been informed by A. Sundarajan understanding of SE [82] (see Related Work). As such, during the interviews we posed questions and attempted to initiate conversations that focused on how StR members look for and post rides, on how StR members build trust, on how they organize the logistics of ride-sharing and how (if) offering reviews works.

In addition to the SE phases listed by [82], the works and accounts presented in our Related Work section [i.e. Solidarity HCI [87], Sharing Cultures [52, 53], Platform Cooperativism [75] also informed the interview guide we created and used. In particular, extrapolating from those accounts we attempted during the interviews to brew up conversations about the activity of sharing in general -beyond ride-sharing as such, the group's sharing norms, the design and management procedures of the group, the future of StR and the role of other institutions (e.g. FB, state) in shaping it.

Our previous experience as active members of StR has been also central throughout the interviewing process and the data analysis accordingly. In particular, it informed the language we have used during the interviews; one contextualized within StR which has to an extent its own 'vocabulary' with which we have been well acquainted. It also affected our stance during the interviews as we occasionally pointed to some well known among StR members events or incidents or internal debates while discussing around different aspects of StR's operation. Similarly, our experience as members and users of StR affected the ways we have interpreted the participants' sayings while performing the Thematic Analysis of which results are presented in the following section.

\section{FINDINGS}

The interviews were held by the first author, either face to face or via VoIP as some of the interviewees were in different locations to the interviewer. Each interview was recorded and lasted between 40 and 70 minutes. In total, the collected data were 510 minutes in duration. They were analysed using Thematic Analysis; a method for identifying, analysing, and interpreting patterns of meaning ('themes') within qualitative data [15]. The collected data were initially coded, a process which led to the creation of the following themes:

'Negotiating ride-sharing', 'Building trust(s)', and 'Appropriating FB to develop a community and a sharing culture'.

\subsection{Negotiating ride-sharing}

In StR, posts 'looking-for' or 'offering' a ride follow the hashtags system we presented in 2.2.2 via which a member indicates the place of departure, the final destination, the ride-share's date or a period of dates when a trip is not date-specified. However, in most occasions, the ride-related posts do not mention the exact time of departure, the amount of monetary contribution, nor a specific route to be followed. Our findings indicate that all those details, as not predefined, are negotiated among members of StR who organize to participate in a ride-share. As our participants reported, those negotiations and agreements occur in a private manner either via personal FB messages or phone calls.

P1 (driver) described the process of setting up the logistics of a ride as a collective process and not a predefined one by those offering the ride and P10, also a driver, mentioned that when they organize a ride-share they usually create a group chat to e-meet each other and discuss the logistics of a ride. Another participant (P9) a non-smoker said that puts no rules in their car beforehand but

"[..] smoking in the vehicle depends on how this is negotiated and what the other persons in the vehicle might want". 
The same person, while discussing the costing of a ride mentioned that divides

"[...] the cost by the number of riders. Except if the car is not full, say, there is only one other person in the car. I will not ask them pay half of the cost. I 'll propose, if they agree, to pay a bit more of what they would if the car was full".

Another participant (P3) mentioned that in the group, occasionally there are some ride offers that do not ask for a monetary contribution and commented

"As no matter what, I' ll do that route, then you are welcome to join. Just be my company. That's really nice, right? That's the general idea, to help each other".

P6, currently a passenger, described a ride they participated in which the driver did not ask for any monetary contribution and while talking about the near future when they will have their own car and offer rides via StR commented

"I wouldn't have any problem if someone would tell me that I cannot contribute that much, I can give you less money or nothing at all".

In the same vein, $\mathrm{P} 2$ mentioned that participates in StR to offer a ride not to

"[..] cut costs [...] sometimes I can take somebody that cannot 'chip-in'[...] they can offer me a coffee, roll some cigarettes or just be a good company"

and also commented

"I don't want to insult someone [...] I do not predefine prices [...] I am not a taxi driver so I leave it on one's hands to decide how much to contribute".

Moreover, our findings indicate that StR members occasionally make posts via which they ask for the transfer of pets or things. In particular, a member driver (P1) narrated a ride-share story, when they transferred a motorbike that belongs to another StR member. Similarly, other participants referred to rides they have participated in where the driver or another passenger were transferring someone else's stuff or pets.

Finally, all participants similarly to P6 underscored that

"Whichever monetary transaction occurs, when occurs, it takes place offline and only in cash at the end of a ride".

Most interviewees did not comment on FB's for-profit nature but described FB as a communication medium that does not make any profit from StR's operation. However, all participants speculated that sooner or later a for-profit ride-sharing company would appear in Greece as well and towards such, while expressing their concerns P1 noted:

"Such a company will change how ride-sharing works as it will impose its very own rules".

Other interviewees (P3, P8) while comparing StR to a for-profit company expressed their content that

" [...] there does not exist an app that would make things more 'rational' and impersonal. [...] I like this type of freedom and self-organization [within StR] [...] if a company appears it's going to set its very own for-profit agenda".

In this light, member passenger (P4) while discussing the process of organizing a ride among StR members described it as one that

"[...] is set up by peers, one to one [...] without the need of any verification from the admin team or somebody else. I have my FB profile, your own and we agree privately as individuals about a ride's logistics. It feels that no one else has a say on this process". 
Another member driver (P7) described ride-sharing within StR both as a self-organized and solidarity-driven practice in the sense that

"[...] people do not want to use public transportation which is a monopoly [in Greece] so they, themselves, have found another way to act and react where they can also sometimes offer a free ride to someone that is not able to chip-in".

In this theme, we presented the interviewees' concerns and thoughts about a scenario in which a for-profit ridesharing company would appear which they described as one that would enforce new rules on how ride-sharing is mediated and performed. On the contrary, we presented our findings which depict organizing the logistics of a ride-share among StR members as a process independent from third parties and as a highly negotiable activity. Moreover, we saw that ride-sharing among StR members is performed as a not-for-profit activity and occasionally solidarity-driven, out of which FB, as the supporting medium is considered to make no direct profit. Finally, as the reviewing system which is suggested by the admin team is not adopted and while extrapolating from our participants narrations via which they described that setting up a ride occurs in private and any monetary transaction is in cash, we observed that organizing ride-shares via StR is a non directly monitored practice.

\subsection{Building trust(s)}

Alongside organizing the logistics of a ride-share, our research participants were prompted to discuss about and reflect on the process/processes of trusting another member to share a ride.

Participants, referred to trusting each other not as fixed, one-off, process but as a "subjective" and contextualized one that traverses different phases. According to P4 (a member of the group who participates as a passenger)

"because of the way StR is structured, everyone decides for himself and with their very own criteria to trust another member of StR or not for a ride-share"

and as reported by another interviewee (P3, the creator and a member of the admin team)

"In StR, everyone has its very own experiences, values and principles. Thus the way I, myself, 'evaluate' and decide might differ from someone else's way".

As already described, the admin team in order to 'facilitate' trust among members of StR has proposed the use of hashtags for the exchange of reviews among StR members. However, as such a more systematized process has not yet been adopted by the group's members, there does not exist one universal reviewing system in place which StR members typically use.

The interviewed drivers and passengers reported that only on very few occasions a potential co-rider asks about the driving skills of a person online; before co-riders meet in person in/on a vehicle. However, P10 (a driver) mentioned that usually their co-riders (passengers) ask them at the very beginning of a ride

"[...] how many years of driving experience I got, what routes I've driven so far [...] I reckon in order to find out if I am an experienced driver or not".

Along the same lines, P7 (driver) mentioned that during a ride, it is typical to be asked "[..] if you 've offered many rides via StR" and also mentioned that

"In order to make my co-riders feel comfortable, I occasionally narrate driving stories which I hope that depict my driving past experience". 
P9, a driver and a member of the admin team while talking about someone's driving skills and reviewing drivers within StR, interestingly underscored that

"When someone has a driving licence, this implies that the state is trusting them to exist on a greek road. I hope that the state knows what it's doing whilst I do not believe so".

While talking about reviewing each other, P10 and P7, both drivers, referred to some occasions when underneath a post offering a ride that they have made, some other members of StR which have traveled with them in the past wrote "on the fly something like a review" in the form of a FB comment which however did not directly refer to their driving skills but to the ride-share in general. Some participants argued that reviewing each other could help the group's operation in the future while P7 presented "good reviews with the use of hashtags" as "clutter" for the group's operation and mentioned that "Bad reviews can be useful, not a 'ton' of good ones". Likewise, members passengers (P4,P5) told us that they most probably wouldn't write a "good review" on StR's page for someone they travelled with but that they would

"[...] definitely report publicly to the rest of the group incidents where drivers might have had a 'weird' behavior towards them".

Moreover, our findings indicate that trust among StR members does not only refer to the driving skills of a person and that someone's good driving skills might not be enough for a StR member to join a ride-share. For example, a member passenger $(\mathrm{P} 4)$ referred to an occasion where they decided to reject an offer from "a possibly professional driver as they were offering empty seats in a freight car". On this incident they mentioned:

"[...] the thought of a girl in the same vehicle with a man that drives a truck, stereotypically caused a sense of insecurity. So, I decided to thank him and let him know that I found another ride".

Similarly, another participant (P8) while talking about why they have decided not to participate in a ride reported:

"[...] something that prevented me from using StR was that the drivers were always men [...] it might be my very personal prejudice but I would feel more comfortable if I was travelling with a driver that was a stranger woman rather than a stranger man"

While talking about the process of trusting each other to organize a ride-share, all participants mentioned that 'scanning' the FB profile of a potential co-rider is a process they tend to go through before agreeing or not to co-travel. This includes visiting a co-rider's FB profile, browsing their uploaded photos, recent posts, videos, pages and groups liked and sometimes checking someone's past activity on StR. P4 (passenger), P1 and P2 (drivers) referred to occasions when they rejected somebody offering a ride due to their FB profile assets which included nationalist symbols. Another participant (P3) referred to FB profiles that they wouldn't contact for a ride-share

\section{"[...] if there was [in a FB profile] any kind of sexist-like posts or information".}

Most participants also stated that the way someone writes a post, writes a personal message or talks on the phone are important factors that affect building trust.

Our interviewees also referred to 'blurry' profiles (those that offer limited personal information), by expressing their concerns while also suggesting potential work-arounds. P3 expressed their anxiety on trusting someone that "do not have any photos on FB or has only few FB friends" and P9 narrated an occasion when they hosted a co-rider

"[...] who had a FB profile with a pseudonym, not a 'real' name and a random photo [...] I explained to them that I cannot travel with someone that I don't know anything about. We chatted and it was [name] who 
studies in [university] and loves to read books. Thus, I told them that there is no problem and I understand that someone wants to stay anonymous online. [...] that everything seems $O K$ and we can travel together".

P1 while referring to such occasions suggested that co-riders can make a video call to "tame this sense of insecurity".

Having mutual friends was a factor which was given importance by the majority of participants (P1,P2,P3,P6,P8) in order to both cope with the occasion of trusting someone with a 'blurry' profile and to trust somebody in general. P1 (driver) referred to an incident when

" [...] a girl who had a photo of 5 cats, sent a message about a ride. We had three mutual friends which live in [name of city]. I then sent a message to one of them and asked about her. [...] later on, when I was informed from this mutual friend that she is $\mathrm{OK}$, I wrote her that we could do the ride together".

Some members drivers (P8, P6) when discussing trust among co-riders underscored that knowing who else is going to be in the vehicle except from the driver can be an important factor that would make them feel more "comfortable" and "relaxed" and P10 (a driver) mentioned that when they organize a ride-share they usually create a group chat.

"I do so in order to let the other passengers know who else is going to be in the car [...] to be able to know via FB who will be the future co-riders and start discussing altogether where we are going, what we want and what we don't want".

In this theme, we presented our findings which indicate that among StR members trusting each other to share a ride is a versatile process, as trust does not only refer to the driving skills of the person who offers a ride, which on some occasions is not seen as the primary factor to join a ride-share. In this regard, we described that building trust among future riders and 'evaluating' a potential co-rider is not a standardized process but is a varying, multilateral and situated operation that takes place in both the digital (i.e. before exchanging personal messages and while organizing the logistics of a ride) and in the physical realm (i.e. while in a ride). This operation is informed by subjective, personal criteria and is affected by factors like the role a member has (i.e. driver, passenger), their gender, the information a potential co-rider offers on their FB profile and a member's digital activity in general, the existence of mutual FB friends, the existence of other people in a vehicle, the ride-share as such. In this theme we also depicted that as primarily FB users, StR members participate in the group with their FB identities ('rich' or 'blurry') which do not always have to coincide their 'real' ones.

\subsection{Appropriating FB to develop a community and a sharing culture}

As also presented in 2.2.2, since 2019, the admin team -except for the admin and creator of the group (P3)- is not always consisted of the same members, as regularly new persons are added and others leave.

In particular, when in 2019, the hashtags system was introduced, the creator of the group and its only admin by that time, asked for help in managing the group's new needs through a video that was posted on the group's wall.

When discussing about this incident, P3 mentioned

"I think that not a lot of members watched that video but I was lucky, as some of those that came across it, expressed their willingness to help. I was also lucky cause those persons were aware of what it should be done and what they need to do and also were motivated to contribute. One of them [name] was already really active before this call and had been sharing with me ideas about the group, thus we already had some online StR-related conversations" 
Since then, other similar calls have been announced in the group's page by members of the admin team and new members have joined while others have left. The members of the admin team we interviewed (P3,P9) reported that they are in charge of managing the group's basic needs. They maintain the group's digital assets (e.g. refresh cover photos, post suggestions in the form of videos for the use and role of the group), they accept new members and moderate the posts made in the group. If a post is not using the hashtags system correctly, the admin team suggests changes. If a post is written in another language, the moderators translate the post also in Greek. If a post is against the ethics/values of the group, it is thus not accepted. Similarly they intervene if a post (despite accepted initially) or a comment are considered to violate the group's rules (i.e. hate speech, bullying speech).

On the later, P3 referred to occasions when members reached them via personal messages to report that "[...] this person did wrote this or that which is sexist or fascist etc)". Accordingly, StR members also use the report option to denounce a post or a comment and "[...] if something is reported 4-5 times then someone from the admin team will most probably intervene" (P3,P9).

Except deleting posts, the admin team participants also referred to occasions when members have been expelled by the group. In specific, P3 described incidents which can lead to such a decision when for example some persons

"repetitively or aggressively [...] don't show respect to some other members and are offensive or impolite or just want to 'import' their doom and gloom or their hatred."

From our interviewees we have been informed that the admin team members do not contribute the same amount of time and effort, as for example there is not something like a shifts' timetable which they decide to follow. Similarly, we were informed and observed that not all admin team members hold the same agency levels. On the latter, P9 told us

"[...] meetings and conversations take place to discuss how we can make StR better, more flexible, more efficient. This 'how-to' is the result of a conversation among the members [of the admin team] that do offer their ideas but at the very end, it is [name of P3] who decides what is going to happen".

While reflecting upon the hashtags system P3 and P9 commented that StR, boldly started to grow in members in 2017. The growing number of participants led to more posts made in the group, thus "to an incremental offer and demand of seats". This meant that more people could find a ride but to do so "they should be able to browse the relevant information" (P3). The increasing number of posts along with FB's 'reality' where on top appear the most popular posts -the ones that have more reactions- and not the most recent ones made more difficult the search and discovery of available rides. This was described as "the main and the 'only' problem" (P3) of the group's performance.

In this regard, during the spring of 2019 and in order to facilitate search and discovery of available seats and overcome "grinding scroll-downs" (P5), a system that makes use of the hashtags was introduced. P3 mentioned that the basic idea of this system was suggested to them by non-members of the admin team. This concept was then firmly designed and introduced gradually to the rest of the group. P9 while commenting on this impromptu search and discovery hashtags system noted:

"[...] if such a codification was made by a professional software engineer that builds a ride-sharing app, the system that they would make, would mandate its users to choose "offering" or "looking-for" from a drop down list, to choose a city from a list, to pick a date from a calendar box [...] but this system was created by amateurs". 
The majority of the interviewees have described the hashtags system as useful and more convenient. However, a member driver (P1) who we interviewed before this system became mandatory, underscored the "fixation of the admin team to use this system" which was articulated in the form of instructive comments made underneath ride-related posts. Similarly, participant passenger (P4) informed us that they are not yet accustomed with that system and that they prefer

"[...] the old 'folksy' way of scrolling down to find a ride. In this way I can also get a glimpse of what has been going on in the group lately".

Participant P9, interestingly highlighted that

"[...] the hashtags system has made the search and discovery of available rides easier without however restricting a group of people like StR to communicate about things other than a ride".

Those "things other than a ride" that $\mathrm{P} 9$ referred to, occur on some occasions in the comments underneath a riderelated post and mainly in non ride-related posts. The later, can be posts via which somebody asks for information about a route or a road trip, posts that raise debates around the operation and the character of the group (e.g. about the costing of rides, about the use of hashtags, about the group's operation during the coronavirus lockdowns), posts made to publicize someone's bad experience in a ride and miscellaneous others (e.g. referring to missing items, offering items like train or plain tickets for free or on a low price), posts like ours which refer to transportation or ride-sharing related research. When asked about those less ride-related posts, the majority of the interviewees reported that since the hashtags system was introduced such posts are not "noisy" and thus they should be allowed but in a moderate way. However, the majority of interviewees suggested that the admin team should intervene by deleting offensive and racist posts or comments. Interestingly, member passenger (P6) while talking about "provocative comments and posts" mentioned

"[...] even when the situation gets out of control it can be 'good' in a way. Through such posts, you can understand few things for the people that are in the group [...] if someone is homophobic, fascist, sexist [...] thus who you should trust or not"

connoting that in this way members of the group that have such views are 'publicly' exposed.

Except for such, many to many online conversations that might occur among StR members, the admin team has twice so far organized offline events in the form of gatherings/parties. During our research, some members of the admin team have been organizing a 10-year party -one we attended- which took place in order to

"[...] bring closer StR members and fans, the community. Even some who might not meet each other on a ride because they most probably have different destinations" (P3).

P2 described StR as a "an experimental and possibly 'educative' process" which can lead to

" [...] the nurturing of a new ideology, another way of life [...] but I'm not sure that this can happen as more and more people of different backrounds join the group".

Similarly, P3,P5 and P9, stated that StR has been already and holds the potential to be a good basis for the propagation of other sharing activities. To this day, a group used for accommodation sharing ("Share the couch") and other local or guild-like ride-sharing (e.g. used by university staff) groups have been created from StR members.

Finally, some interviewees also mentioned that in some occasions in order to set up a ride-share they have completely bypassed StR; they did not make an 'offering' or 'looking-for' public post but directly contacted some StR members with which they have shared a ride in the past. 
In this theme, we stated how the admin team of StR is an internal body -in the sense that its members use themselves StR and participate in ride-shares- composed of different persons who help in the management, sustainment and evolution of the group -which however do not hold the same levels of agency. We also presented how the scaling up of StR complicated the search and discovery of available ride-shares, a condition which led to the 'design' and development of an impromptu system, one that did not ask for professional programming skills. We also reported that among StR members new sharing initiatives have been developed. Finally, we presented how building relations, which undoubtedly affects building trust(s) among StR members, are facilitated by offline meetings and collective online discussions which do not always refer to the activity of ride-sharing per se.

\section{DISCUSSION}

In our Findings section, we brought to the surface the plural ways that StR members build trust(s), the negotiations that occur among individuals when setting-up a ride-share and the many-to-many discussions that take place among StR users as members of a broader community of sharers. We also described how the medium (FB) has been appropriated by StR members to first and foremost initiate a ride-sharing 'platform' on top of a medium that has not been designed for the sharing of physical goods/resources per se, to facilitate ride-sharing as a service among individuals and to enhance the performance of StR as an internally managed, community-driven and participatory SE initiative.

We believe that the sociotechnical processes as described above that manifest in StR that stand in contrast to the operation of the 'big' platform-firms, which are developed in ways that occasionally design out participation and self-organization [52, 75, 76], can be informative in our 'Solidarity HCI-driven' inquiry for the development of community-driven SEPs outside FB or platforms of a similar ilk.

Arguably however, someone could ask why we need and suggest the development of other infrastructures, if groups like StR can operate -and to an extend successfully- on already existing social media platforms. We believe T.Scholz's concept of Platform Cooperativism is key to answering such a practical query. Within Platform Cooperativism, T.Scholz's [75] proposes a collective type of ownership of SEPs, argues in favour of transparent data and suggests that SEPs' users should participate in the design decisions.

Grounding T.Scholz's suggestions in StR and the need for the development of other infrastructures, we need to underscore that FB (in contrast to some of our participants' views) is a for-profit platform -while not directly profiting from ride-shares organized via StR (yet)- where users' data become the product that is sold to third-parties which use them for different, mainly for-profit, reasons[33].

In addition, we need to boldly state the manifold and precarious dependence of StR on FB in spite of the fact that so far it has favored to an extend its operation. This manifold and precarious dependence can be exemplified and illustrated in various situations. For example, in the scenario that access to FB is blocked [7] then also StR as all FB groups will have to pause their operation as such. Or in the expected scenario that FB deploys their bespokely designed ride-sharing app [23] which could arguably affect StR or even lead to its permanent close-down. Among other occasions, we can speculate that future changes in the affordances of FB groups could make StR's impromptu search and discovery system idle, something that could possibly heavily affect the group's operation.

In this regard, while on the one hand we acknowledge the novelty of StR as a community-driven SE 'platform' on the other hand and for the reasons we mentioned above, we believe that such initiatives could be better 'nested' in other, more autonomous and independent, infrastructures which are more transparent and better designed to support community building and self-organization.

Manuscript submitted to ACM 
As a result, we suggest malleability as a design quality of the SEPs we are hereby discussing. We see malleability evidenced in design affordances which can better support agency over a sharing activity, as to what can be shared, to the digital designs used to support sharing activities, to building trust(s) and identities. We also see malleability as a design quality that can favor the development of a generative sharing culture. In addition, we propose the employment of trust building mechanisms which aim at building relational trust that is evidenced in enduring and resilient relations. Finally, we suggest that within the aim of developing familiarity and authentic relations, SEPs should employ designs that as non-normalizing avoid the reproduction of stereotypes and thus favor users' self-determination.

\subsection{Malleability as a design attribute of SEPs}

Malleability (or alternatively referred to as tailorability and appropriability) as a design quality of IT artefacts have been intensively investigated by HCI and CSCW research [54, 69] especially within the field of End-User Development(EUD). EUD refers to a set of methods, techniques and tools to create, modify or extend an IT artefact without always having professional programming skills [68]. Among EUD researchers it has been predicted [30] that HCI design goals, if aimed at empowering end users, will evolve from easy-to-use to easy-to-adapt and develop. Grounding this in the design of platforms to support the SE, user and community empowerment has always been articulated as a main point of departure and a key design goal [75-77].

Grounding malleability in our findings from researching StR, we observed that FB, as a 'malleable', i.e. adaptable and flexible (in the context we have been exploring it, i.e. ride-sharing) communication and social media infrastructure, played a key role in StR's emergence and its development eventually into a widely used and community-driven ride-sharing 'platform'.

More specifically, as we previously described, despite the fact that FB groups are not designed to support sharing of physical resources/goods per se, FB afforded the initial plan of a user (the creator of StR) to set up a rudimentary ride-sharing group where they, some of their friends and some friends of friends could exchange information in order to ultimately organize ride-shares. In addition, we saw that when the group got congested as more and more people have started using it and members were not able to review relevant options in a timely manner [51] some members of StR 'designed' a process to facilitate search and discovery of available rides which was materialized as a 'bricolage of hashtags'.

Similarly, we saw that a similar hashtags' system has been proposed as a means of giving feedback aiming at supporting trust-building among StR members. Both of these impromptu designs were contributed and developed by members of the community itself. Developments which as we presented did not ask for professional programming skills and most importantly they were afforded by FB.

Moreover, we found that the introduction and application of hashtags -which we interpret as a hint of malleability in the FB context we have been researching- has been a key factor for the sustainment of StR as a participatory scheme where except traditional peer-to-peer ride-sharing evidenced in posts of 'offering' or 'looking for' rides, its members exchange other information (e.g. about road trips, about routes, train or plain ticket giveouts), discuss about StR's character and performance (e.g. posts about the costing of rides, posts introducing designs suggestions/ideas), engage with the group's management (i.e. posts explaining the hashtags system, asking for volunteers) and future (e.g. during the recent lockdown) etc. Such many-to-many discussions have made StR except for a peer-to-peer platform also an open 'forum' space. One of which operation, we believe is favouring participation, community building, the development of a collective identity and the propagation of sharing in general. As we found, the latter, is evidenced in the scaling out 
[55] of StR as some of its members have created new sharing initiatives within FB groups that operate in similar ways (e.g. an accommodation-sharing group, local-ride sharing groups).

Departing from the above interpretations and the works that have shaped our HCI lens[52, 75, 87], we suggest malleability as a design feature of the SEPs we have been interested in discussing.

To the best of our knowledge there have not (yet) been developed SEPs that employ malleability as a design feature. We think however, that social media infrastructures, as infrastructures adaptable to SE contexts (i.e. in cases like ride-sharing as in StR and in freecycling groups [62]) can be informative towards that design approach.

Similarly, we can see elements of malleability in the so-called project management apps despite them being designed to support mainly cooperation and the management of projects among co-workers. In such apps, users can create multiple groups/projects, allocate tasks, make wall-posts and participate in many-to-many discussions and they can to an extend customize some of the apps' features. While such are not designed to mediate processes like the ones typically manifested within SE (i.e. search and discovery of resources, building trust, organizing logistics) we believe that they have affordances which can be instructive for the development of malleable SEPs.

To better ground malleability in SE, we envision malleable SEPs as 'bedrock' communication infrastructures on top of which multiple and different SE groups can be developed. As a result, we picture that these communication infrastructures, afford the creation of ride-sharing groups along with room-sharing, food-sharing and tool-sharing ones etc. We also see malleability as the ability of SE groups' users to adjust their sharing activities during crises (e.g. socioeconomical, pandemics, wildfires etc). For example we picture that members of a ride-sharing group, instead of pausing their activity due to a lockdown or a wildfire, can modify the design of the group and transform it to one that can support those in need (e.g. drivers can deliver food to those that are quarantined) or to a group that can be used to organize ride-shares that get people away from the region in danger or bring in volunteers. Such transformations are also in alignment with work on solidarity movements, which as reported in [87] have the capacity to adjust and evolve based on situated human needs and collective priorities.

Moreover, our findings which have indicated the key role that StR's 'forum' have played in the evolution of the group as a community, point to the need for malleable SEPs to offer (possibly though a template-like form) a series of tools that end-users of each SE group can use to create forums or smaller chat rooms. Similarly, departing from the case of StR where the supporting medium (FB), afforded the bottom-up creation of a search and discovery system we point to the importance of developing designs that afford customizations by end users that can better support a specific sharing activity.

To be more specific, a search and discovery mechanism to support a ride-sharing SE context, where locations and dates are key information, can differ considerably from a context where members of a SE group practice foodsaving and foodsharing or share tools. In the latter, date and location can be important but also important for members, would be to browse and post information on the type of a tool, its availability (i.e. for how long it can be borrowed), its current holder (in the occasion that tools are community owned and change hands in a fast pace), its need to be repaired from a member of the community that has the skills etc. Arguably, the hashtags system as deployed in StR afforded by FB, could be adapted for the search and discovery of a lot of different types of shareable resources. In that, we do not suggest the employment of a hashtags system as such, but that malleable SEPs should offer indexing tools that can be picked out possibly from a tools' library which can serve various purposes across multiple types of resources being shared.

However, we note that to favor community building and mutual understanding, such tools should not be designed for the standardization of a sharing activity but should operate to supplement sharing as a social practice where negotiations take place [52]. This view can be better illustrated by the case of StR where we found that the hashtags' Manuscript submitted to ACM 
system did better organize ride-related posts, as to date of departure, starting and ending destination, and facilitated the matchmaking of ride-sharers withought however enforcing a high level of standardization, one that could be materialized for example in the use of formal fields -through which drivers could except date and destination, predefine in-vehicle rules, predefine the route to be followed, the cost per person etc - or in a 'book-now' option, which can be found in other SE platforms (i.e. Blablacar). In this regard, we believe that such mechanisms that can turn sharing into a highly technologized processes should not be opted by the platforms we discuss here as removing negotiations can severely affect sharing as a community-driven and potentially caring-based and solidarity-driven activity[52].

Overall, in this discussion point, we have proposed malleability as design attribute of SE platforms, one which we described as the ability that SE users have to create and participate in multiple SE groups and to adjust those groups' designs and processes to better support varying sharing activities. With agency, community needs, mutual understanding and solidarity in mind, we similarly stated the need for the deployment of mechanisms that afford the development of sociotechnical processes that can support different types of sharing activities which however do not standardize sharing but aim at favouring negotiations.

\subsection{Relational trust and self-determined identities}

Trust, as a key element of sharing practices among 'strangers', is one of the most researched topics within the HCI community $[6,41,49,57]$. Building trust has been described as of paramount importance for the operation of SEPs in order to mitigate risk among sharers [14, 82]. To support trust building, rating and reputation systems have been widely adopted as quick fixes, simplifying the peer-to-peer exchange of resources and services. Even though such rating, filtering and reputation schemes are useful, especially when trust-building needs to work in a large scale, they have been widely criticized from multiple fronts, for example in relation to how they affect working conditions for gig economy workers and whether they are actually effective [75, 76]. Similarly, such trust-building and risk-averse schemes have been criticized for standardizing and objectifying the process of getting to know each other and trusting each other, for removing negotiations among sharers [52] and for prompting their users to create 'generic' digital identities [26]. Except this one-off and 'superficial' type of trust, building relational trust which is of high relevance to our work, has been introduced as a different approach. Relational trust refers to the trust that is built slowly and progressively over repeated interactions among individuals [19]. We believe that such repeated interactions can create new bonds and enduring relations, thus favor community building, and lead to more resilient SE schemes.

Within the infrastructures described above as malleable 'bedrock' platforms which afford that its members can create and participate in multiple SE groups we see the potential for relational trust to manifest. As such, we can picture that members of a ride-sharing group built on top of a malleable SEP, join the platform initially in order to participate in typical ride-sharing while progressively use it to organize other sharing activities; for example, group road-trips which could bring members together for longer periods and in more intimate settings. In a similar scenario, within a group developed to facilitate tools' sharing we can picture its members building relations thus familiarity, while from typical tool-sharing, they progressively use the platform to organize repair cafes, 'building and mending parties' etc. Moreover, within this continuity where a slow proliferation of sharing manifests, we can picture that members already familiar and connected to each other in a context (i.e. ride-sharing, tool-sharing) can segue into other sharing contexts (e.g. from ride-sharing and group-road trips to accommodation-sharing) or create solidarity sharing groups in cases of crises as described before. We argue that relational trust is important as a design goal as it enables people to develop meaningful and potentially more resilient relations and as such engage outside of the confinements of a very specific SE group and a very specific SE activity. 
To better support the development of familiarity, we also suggest that in those SEPs, users should be offered digital tools via which they can delegate trust among each other. In this suggestion we also reflect on our findings from researching StR (as also found in [37]), which indicated how having mutual friends on FB and 'real word' acquaintances worked as a referral chain for StR members, with people being able to ask their mutual friends to 'vouch' for a future co-rider. In this regard, we suggest that users can join one or another SE group provided that a number of active members support their admission. Such a number cannot be a fixed one but should be adjustable to each SE group depending on the sharing activity that is performed, the size of the group etc. This way of vouching for somebody comes in contrast to the mechanisms that are employed in some SEPs where trust is in tandem with the abovedescribed 'crisp' reviewing and rating systems [52] supported by some third-party verifications. Such vouching processes promoted as an extra layer of trust to enhance users' eligibility and trustworthiness appear in various platforms where users are prompted to upload a screenshot of their government-issued ID of which status is checked by the third-party collaborating firm.

In this transition from trusting each other based on highly technologized processes and external vouching, to building familiarity through relational trust as the result of repeated interactions, we similarly see the opportunity and the requirement for members of SE groups to participate with more self-determined and 'authentic' identities rather than government-issued or 'real' ones. We think that such design affordances can favor pluralism and avoid the reproduction of stereotypes. As we saw in our findings, operating a ride-sharing scheme like StR on a social media platform like FB allowed users to construct, present and narrate more self-determined identities. More specifically, despite the fact that as any medium, FB introduces its own sociotechnical limitations for the construction of identities [38, 43, 64], we saw that StR members participate in the group with identities that are explicit or implicit (in relation to gender, connection to real identity or name, etc.), something that is known as a common and accepted practice in most social networking platforms. Such rich identities also materialise the plural trust mechanisms that we have discussed, as they enable users to reflect on these self-determined and narrated (on FB) identities before making a decision about with whom and what can be shared. For members of StR the availability of these identities on FB posed dilemmas which are not typically found on SEPs, their matchmaking and reviewing systems (i.e. would you share a ride with a nationalist or a climate change denier?) that nonetheless allowed them to make a more informed and personal decision rather than one based on an aggregation of scores or a voucher offered by a firm.

Those findings point to the limitations of users' profiles on existing SEPs that in most occasions reduce peoples' identities to a name, a picture and a rating. Thus, they inform our suggestion that SEPs as platforms aiming at building relational trust among its members that is evidenced in authentic and inclusive relations, should employ mechanisms that can expand the reach of legal identities [25] and favor users self-affirmation instead of prompting users to enhance their trustworthiness (or marketability) by creating generic profiles that tell a right and safe story [26].

Having said that, we should also note that not all SEPs should (or could) construct FB-like, data and media rich digital profiles for their users, and there are multiple and important reasons (e.g. privacy) to resist such a development that might lead to arbitrarily and naively 'rating' people based on their online self-constructed or analytics-driven identities. To mitigate such phenomena, we believe that vouching mechanisms as the ones presented above are towards the right direction.

From summarizing the above, we argue that SEPs should invest in the development of mechanisms that can support building relational trust and enduring resilient connections instead of aiming at increasing an 'impersonal' and fast sharing. In addition, we have argued that SEPs designs should avoid reproducing the modernist and normalizing views of our current society [53] and thus enable openness, plurality and self-determination [80]. 


\section{LIMITATIONS}

Reflecting on the process we have followed to engage with StR members, we acknowledge that the individuals who did not provide adequate contact details via our questionnaire, despite their willingness to participate, have been excluded from our research. Moreover, as during this process we perceived and positioned ourselves as both HCI researchers and active members of StR's community and we thus chose -which was also technically feasible- to directly (on the group's 'forum') express our research intentions and though a public 'open call' to look for participants (instead of for example contacting all members of StR in private) we acknowledge the possibility that some StR members never came across our posts (e.g. because at that time they did not check StR's activity or because they have not been using FB for some time etc) and the possibility that some members saw our first post but not our questionnaire. We finally acknowledge that some individuals -technically still members of StR- which might have stopped using the group due to various reasons (e.g. because they are using ride-sharing alternatives, because they might have been disappointed by StR's operation or ride-sharing in general) might also have not encountered our research related posts.

Thus, we understand as a limitation that as in any qualitative HCI study of this type, some voices are not or might not be fully represented in this work. However, we believe that the approach we have followed have amply supported our aim to gather insights into the sociotechnical processes that manifest within a community-driven ride-sharing initiative and building upon those insights to provide design implications relevant to CSCW and HCI for the development of SEPs.

\section{CONCLUSION}

In response to a lack of research on SE that promotes community building, participation and a caring-based sharing in this paper we presented our findings from our engagement with a ride-sharing initiative that emerged in a FB group and has developed into a community-driven SE 'platform'. Our findings pointed to the multiple ways and mechanisms through which the group members build trusts and social relations and to the negotiations that manifest both among individual riders and among StR users as members of a community. Moreover, we presented how they have appropriated FB to support ride-sharing as a service and the group's operation in general. Finally, we observed that the researched FB group except for ride-sharing purposes is also used to serve other sharing activities and that new sharing communities have emerged from this group. Departing from our findings from researching StR, in our discussion, we suggested malleability as a design quality of SEPs that can favor self-organization, community building and participation and the development of a generative sharing culture. We also suggested the employment of designs which aim at supporting the establishment of relational trust and we argued for the employment of designs which can foster pluralism and self-affirmation.

We acknowledge that through this work we have engaged with malleable SEPs on their very embryonic form and thus we argue that more work (e.g. research or pilot projects) should be performed in order to explore potentialities (e.g. data ownership models, collaborative designing and tailoring, governance models) and other issues (e.g. managing conflicts, scale, privacy) of which we have not been able to do in this paper. However, we see this work as a small contribution in the 'sharing discourse' that points towards reclaiming SE as one that can favor sharing as a community-driven, self-organized and potentially altruistic activity. 


\section{ACKNOWLEDGMENTS}

We would like to thank all StR members who have shared their experiences, concerns, comments and thoughts during the undertaken interviews or under less formal circumstances. We are particularly grateful for the assistance and advice given during this research by Al James, Professor of Economic Geography in Newcastle University. We would also like to thank Alexandra Tzanidou and Sami Abosaleh, HCI researchers from Open Lab Newcastle for sharing their comments and ideas throughout this process. This research was funded by EPSRC's Center for Doctoral Training in Digital Civics (EP/L016176/1) and partially by the EPSRC's Digital Economy Research Center (EP/M023001/1) (the second author's Research Associate position at the time).

\section{REFERENCES}

[1] Jonathan P. Allen. 2016. The Sharing Economy: Studying Technology-Mediated Social Movements. In Proceedings of the 2016 ACM SIGMIS Conference on Computers and People Research (Alexandria, Virginia, USA) (SIGMIS-CPR '16). Association for Computing Machinery, New York, NY, USA, 65-67. https://doi.org/10.1145/2890602.2890609

[2] Alberta Andreotti, Guido Anselmi, Thomas Eichhorn, Christian Pieter Hoffmann, Sebastian Jürss, and Marina Micheli. 2017. European perspectives on participation in the sharing economy. (2017). https://ssrn.com/abstract=3046550

[3] Athina Arampatzi. 2018. Constructing solidarity as resistive and creative agency in austerity Greece. Comparative European Politics 16, 1 (2018), 50-66.

[4] M. Avital, J. Carroll, A. Hjalmarsson, N. Levina, A. Malhotra, and A. Sundararajan. 2015. The Sharing Economy: Friend or Foe?. In ICIS.

[5] Gabriela Avram, Jaz Hee-jeong Choi, Stefano De Paoli, Ann Light, Peter Lyle, and Maurizio Teli. 2017. Collaborative economies: From sharing to caring. In Proceedings of the 8th International Conference on Communities and Technologies. 305-307.

[6] Natã M. Barbosa, Emily Sun, Judd Antin, and Paolo Parigi. 2020. Designing for Trust: A Behavioral Framework for Sharing Economy Platforms. In Proceedings of The Web Conference 2020 (Taipei, Taiwan) (WWW'20). Association for Computing Machinery, New York, NY, USA, $2133-2143$. https://doi.org/10.1145/3366423.3380279

[7] Luis Fernando Barón. 2012. More than a Facebook revolution: Social movements and social media in the Egyptian Arab Spring. (2012).

[8] Kyle Barron, Edward Kung, and Davide Proserpio. 2018. The Sharing Economy and Housing Affordability: Evidence from Airbnb. In Proceedings of the 2018 ACM Conference on Economics and Computation (Ithaca, NY, USA) (EC '18). Association for Computing Machinery, New York, NY, USA, 5. https://doi.org/10.1145/3219166.3219180

[9] Arianne Renan Barzilay and Anat Ben-David. 2016. Platform inequality: gender in the gig-economy. Seton Hall L. Rev. 47 (2016), 393.

[10] Russell Belk. 2014. You are what you can access: Sharing and collaborative consumption online. Fournal of business research 67, 8 (2014), $1595-1600$.

[11] Yochai Benkler. 2004. Sharing Nicely: On Shareable Goods and the Emergence of Sharing as a Modality of Economic Production. The Yale Law Journal 114 (11 2004), 273. https://doi.org/10.2307/4135731

[12] Michelle Birdsall. 2014. Carsharing in a sharing economy. Institute of Transportation Engineers. ITE Journal 84, 4 (2014), 37.

[13] Marco Böckmann. 2013. The Shared Economy: It is time to start caring about sharing; value creating factors in the shared economy. University of Twente, Faculty of Management and Governance (2013).

[14] Rachel Botsman and Roo Rogers. 2011. What's mine is yours: how collaborative consumption is changing the way we live. Vol. 5. Collins London.

[15] Virginia Braun and Victoria Clarke. 2006. Using thematic analysis in psychology. Qualitative Research in Psychology 3, 2 (2006), 77-101. https: //doi.org/10.1191/1478088706qp063oa arXiv:https://www.tandfonline.com/doi/pdf/10.1191/1478088706qp063oa

[16] Mark W Burris and Justin R Winn. 2006. Slugging in Houston-casual carpool passenger characteristics. Fournal of Public Transportation 9, 5 (2006), 2.

[17] Mingming Cheng and Carmel Foley. 2018. The sharing economy and digital discrimination: The case of Airbnb. International fournal of Hospitality Management 70 (2018), 95-98.

[18] Daniel G Cockayne. 2016. Sharing and neoliberal discourse: The economic function of sharing in the digital on-demand economy. Geoforum 77 (2016), 73-82.

[19] Jerome Cranston. 2011. Relational trust: The glue that binds a professional learning community. Alberta journal of educational research 57, 1 (2011), $59-72$.

[20] Nestor M Davidson and John J Infranca. 2015. The sharing economy as an urban phenomenon. Yale L. \& Pol'y Rev. 34 (2015), 215.

[21] Valerio De Stefano. 2015. The rise of the just-in-time workforce: On-demand work, crowdwork, and labor protection in the gig-economy. Comp. Lab. L. \& Pol'y f. 37 (2015), 471.

[22] Tawanna R. Dillahunt and Amelia R. Malone. 2015. The Promise of the Sharing Economy among Disadvantaged Communities. Association for Computing Machinery, New York, NY, USA, 2285-2294. https://doi.org/10.1145/2702123.2702189

[23] Jillian D’Onfro. 2016. Facebook has a clever idea to turn itself into a ride-sharing platform. Retrieved March 14, 2020 from https://www.businessinsider. com/facebook-event-based-ride-sharing-2016-1

Manuscript submitted to ACM 
[24] Paul Dourish. 2010. HCI and Environmental Sustainability: The Politics of Design and the Design of Politics. In Proceedings of the 8th ACM Conference on Designing Interactive Systems (Aarhus, Denmark) (DIS '10). Association for Computing Machinery, New York, NY, USA, 1-10. https://doi.org/10.1145/1858171.1858173

[25] Paul Dunphy and Fabien AP Petitcolas. 2018. A first look at identity management schemes on the blockchain. IEEE Security \& Privacy 16, 4 (2018), 20-29.

[26] Benjamin Edelman, Michael Luca, and Dan Svirsky. 2017. Racial Discrimination in the Sharing Economy: Evidence from a Field Experiment. American Economic fournal: Applied Economics 9, 2 (April 2017), 1-22. https://doi.org/10.1257/app.20160213

[27] Marcus Felson and Joe L Spaeth. 1978. Community structure and collaborative consumption: A routine activity approach. American behavioral scientist 21, 4 (1978), 614-624.

[28] Anny Fenton. 2013. Making markets personal: Exploring market construction at the micro level in the car-sharing and time bank markets. Unpublished paper, Harvard University (2013).

[29] Erik Ferguson. 1997. The rise and fall of the American carpool: 1970-1990. Transportation 24, 4 (1997), 349-376.

[30] Gerhard Fischer and Elisa Giaccardi. 2006. Meta-design: A framework for the future of end-user development. In End user development. Springer, 427-457.

[31] Russell Freedman. 2006. Freedom walkers: The story of the Montgomery bus boycott. Holiday House.

[32] Koen Frenken and Juliet Schor. 2019. Putting the sharing economy into perspective. In A Research Agenda for Sustainable Consumption Governance. Edward Elgar Publishing.

[33] Christian Fuchs. 2012. The political economy of privacy on Facebook. Television \& New Media 13, 2 (2012), 139-159.

[34] Masabumi Furuhata, Maged Dessouky, Fernando Ordóñez, Marc-Etienne Brunet, Xiaoqing Wang, and Sven Koenig. 2013. Ridesharing: The state-of-the-art and future directions. Transportation Research Part B: Methodological 57 (11 2013), 28-46. https://doi.org/10.1016/j.trb.2013.08.012

[35] Athanasios Galanis, George Botzoris, Anastasios Siapos, Nikolaos Eliou, and Vassilios Profillidis. 2017. Economic crisis and promotion of sustainable transportation: A case survey in the city of Volos, Greece. Transportation Research Procedia 24 (12 2017), 241-249. https://doi.org/10.1016/j.trpro. 2017.05.114

[36] Viola Gaskell. 2019. Hong Kong Protesters Have Created Their Own Uber to Keep Themselves Safe. Retrieved March 20,2020 from https://www.vice. com/en_in/article/a359zz/hong-kong-protesters-create-own-uber-safe

[37] Sunil Ghelawat, Kenneth Radke, and Margot Brereton. 2010. Interaction, Privacy and Profiling Considerations in Local Mobile Social Software: A Prototype Agile Ride Share System. In Proceedings of the 22nd Conference of the Computer-Human Interaction Special Interest Group of Australia on Computer-Human Interaction (Brisbane, Australia) (OZCHI '10). Association for Computing Machinery, New York, NY, USA, 376-379. https: //doi.org/10.1145/1952222.1952307

[38] Oliver L Haimson, Jed R Brubaker, Lynn Dombrowski, and Gillian R Hayes. 2015. Disclosure, stress, and support during gender transition on Facebook. In Proceedings of the 18th ACM Conference on Computer Supported Cooperative Work \& Social Computing. 1176-1190.

[39] Juho Hamari, Mimmi Sjöklint, and Antti Ukkonen. 2016. The sharing economy: Why people participate in collaborative consumption. Fournal of the Association for Information Science and Technology 67, 9 (2016), 2047-2059. https://doi.org/10.1002/asi.23552 arXiv:https://asistdl.onlinelibrary.wiley.com/doi/pdf/10.1002/asi.23552

[40] Pierre Hausemer, Julia Rzepecka, Marius Dragulin, Simone Vitiello, Lison Rabuel, Madalina Nunu, Adriana Diaz Rodriguez, Emma Psaila, Sara Fiorentini, Sara Gysen, et al. 2017. Exploratory study of consumer issues in online peer-to-peer platform markets. European Commission (EUR 2017.4058 EN) (2017).

[41] Florian Hawlitschek, Timm Teubner, Marc Thomas Philipp Adam, Nils S Borchers, Mareike Moehlmann, and Christof Weinhardt. 2016. Trust in the sharing economy: An experimental framework. (2016).

[42] Katherine Hoffmann, Panos Ipeirotis, and Arun Sundararajan. 2016. Ridesharing and the use of public transportation. (2016).

[43] Amanda Holpuch. 2015. Native American activist to sue Facebook over site's 'real name' policy. Retrieved December 2, 2020 from https://www. theguardian.com/technology/2015/feb/19/native-american-activist-facebook-lawsuit-real-name

[44] Keren Horn and Mark Merante. 2017. Is home sharing driving up rents? Evidence from Airbnb in Boston. fournal of Housing Economics 38 (2017), $14-24$

[45] Anthony Kalamar. 2013. Sharewashing is the new greenwashing. OpEd News (2013).

[46] Hanna Kallio, Anna-Maija Pietilä, Martin Johnson, and Mari Kangasniemi. 2016. Systematic methodological review: developing a framework for a qualitative semi-structured interview guide. Journal of advanced nursing 72, 12 (2016), 2954-2965.

[47] Qing Ke. 2017. Sharing Means Renting? An Entire-Marketplace Analysis of Airbnb. In Proceedings of the 2017 ACM on Web Science Conference (Troy, New York, USA) (WebSci '17). Association for Computing Machinery, New York, NY, USA, 131-139. https://doi.org/10.1145/3091478.3091504

[48] Esther Keymolen. 2013. Trust and technology in collaborative consumption. Why it is not just about you and me. Bridging distances in technology and regulation 135 (2013), 135-150.

[49] Svetlana Kim and Yong-Ik Yoon. 2014. Recommendation system for sharing economy based on multidimensional trust model. Multimedia Tools and Applications 75 (12 2014), 1-14. https://doi.org/10.1007/s11042-014-2384-5

[50] Christopher Koopman, Matthew Mitchell, and Adam Thierer. 2014. The sharing economy and consumer protection regulation: The case for policy change. F. Bus. Entrepreneurship \& L. 8 (2014), 529. 
[51] Airi Lampinen and Barry Brown. 2017. Market Design for HCI: Successes and Failures of Peer-to-Peer Exchange Platforms. Association for Computing Machinery, New York, NY, USA, 4331-4343. https://doi.org/10.1145/3025453.3025515

[52] Ann Light and Clodagh Miskelly. 2015. Sharing economy vs sharing cultures? Designing for social, economic and environmental good. Interaction Design and Architecture (s) 24 (2015), 49-62.

[53] Ann Light and Clodagh Miskelly. 2019. Platforms, Scales and Networks: Meshing a Local Sustainable Sharing Economy. Comput. Supported Coop. Work 28, 3-4 (June 2019), 591-626. https://doi.org/10.1007/s10606-019-09352-1

[54] Alessio Malizia, Stefano Valtolina, Anders I. Mørch, Alan Serrano, and Andrew Stratton (Eds.). 2019. End-User Development - 7th International Symposium, IS-EUD 2019, Hatfield, UK, July 10-12, 2019, Proceedings. Lecture Notes in Computer Science, Vol. 11553. Springer. https://doi.org/10. 1007/978-3-030-24781-2

[55] Ezio Manzini. 2015. Design, when everybody designs: An introduction to design for social innovation. MIT press.

[56] Laura Forlano Maryam Heidaripour. 2016. A Feminist Perspective on the Future of Sharing Platforms. Retrieved March 25, 2020 from https: //futureofplatforms.wordpress.com/position-papers/

[57] Frédéric Mazzella, Arun Sundararajan, V Butt d'Espous, and Mareike Möhlmann. 2016. How digital trust powers the sharing economy. IESE Business Review 26, 5 (2016), 24-31.

[58] Ben Moore. 2018. Social media 'lifts' are becoming an 'illegal industry'. Retrieved March 2, 2020 from https://www.bbc.com/news/av/uk-englanddorset-46048914/social-media-lifts-are-becoming-an-illegal-industry

[59] Tatiana Moschovou and Yannis Tyrinopoulos. 2018. Exploring the effects of economic crisis in road transport: The case of Greece. International fournal of Transportation Science and Technology 7, 4 (2018), 264-273.

[60] Konstantinos Mpogas, Pantelis Kopelias, Lampros Mitropoulos, and Konstantinos Kepaptsoglou. 2017. Road Safety in urban areas in Greece during economy downturn. A before-after comparison. Transportation research procedia 24 (2017), 228-234.

[61] Evanthia Nanaki. 2018. Measuring the Impact of Economic Crisis to the Greek Vehicle Market. Sustainability 10, 2 (2018), 510.

[62] Lukas Norbutas and Rense Corten. 2018. Sustainability of generalized exchange in the sharing economy: the case of the "freecycling" Facebook groups. International fournal of the Commons 12, 1 (2018), 111-133.

[63] Ministry of Environment Energy and Climate Change Greece. 2016. Sustainable Mobility Aims. Retrieved Dec 2, 2020 from http://www.ypeka.gr/ Default.aspx?tabid=628\&language $=\mathrm{el}-\mathrm{GR}$

[64] Sunjoo Oh and Jae Yun Moon. 2016. Calling for a Shared Understanding of the "Sharing Economy". In Proceedings of the 18th Annual International Conference on Electronic Commerce: E-Commerce in Smart Connected World (Suwon, Republic of Korea) (ICEC '16). Association for Computing Machinery, New York, NY, USA, Article 35, 5 pages. https://doi.org/10.1145/2971603.2971638

[65] Craig N Oren. 1998. Getting Commuters Out of Their Cars: What Went Wrong. Stan. Envtl. Lf 17 (1998), 141.

[66] Oleg Pachenkov and Alina Yashina. 2017. When Sharing Economy Meets Digital One: Towards Understanding of New Economic Relations. In Proceedings of the Internationsl Conference on Electronic Governance and Open Society: Challenges in Eurasia (St. Petersburg, Russia) (eGose '17). Association for Computing Machinery, New York, NY, USA, 91-98. https://doi.org/10.1145/3129757.3129774

[67] Panos Papaioannou and Christiana Konstantinidou. 2011. Restructuring the public transport network in times of financial crisis: Solutions from Thessaloniki. Retrieved March 2, 2020 from https://slideplayer.com/slide/6943588/

[68] Fabio Paternò and Volker Wulf. 2017. New Perspectives in End-User Development. Springer.

[69] Volkmar Pipek, Mary-Beth Rosson, and Volker Wulf. 2009. End-User Development: 2nd International Symposium, IS-EUD 2009, Siegen, Germany, March 2-4, 2009, Proceedings. Vol. 5435. Springer.

[70] Theodoros Rakopoulos. 2014. Resonance of solidarity: meanings of a local concept in anti-austerity Greece. Fournal of Modern Greek Studies 32, 2 (2014), 313-337.

[71] Daniel Rauch and David Schleicher. 2015. Like Uber, but for local governmental policy: the future of local regulation of the sharing economy. George Mason Law \& Economics Research Paper 15-01 (2015).

[72] Lizzie Richardson. 2015. Performing the sharing economy. Geoforum 67 (2015), 121-129.

[73] Alvin E Roth. 2015. Who gets what-and why: The new economics of matchmaking and market design. Houghton Mifflin Harcourt.

[74] Naomi Schoenbaum. 2016. Gender and the sharing economy. Fordham Urb. Lf 43 (2016), 1023.

[75] Trebor Scholz. 2016. Platform cooperativism. Challenging the corporate sharing economy. New York, NY: Rosa Luxemburg Foundation (2016).

[76] Juliet Schor. 2014. Debating the sharing economy. Retrieved Nov 1, 2020 from https://greattransition.org/publication/debating-the-sharing-economy

[77] Juliet B Schor and William Attwood-Charles. 2017. The "sharing" economy: labor, inequality, and social connection on for-profit platforms. Sociology Compass 11, 8 (2017).

[78] Juliet B Schor, Will Attwood-Charles, Mehmet Cansoy, Isak Ladegaard, and Robert Wengronowitz. 2018. Dependence and precarity in the sharing economy. Unpublished Paper, Boston College (2018).

[79] Tom Slee. 2017. What's yours is mine: Against the sharing economy (2nd ed.).

[80] Katta Spiel, Os Keyes, Ashley Marie Walker, Michael A. DeVito, Jeremy Birnholtz, Emeline Brulé, Ann Light, Pınar Barlas, Jean Hardy, Alex Ahmed, Jennifer A. Rode, Jed R. Brubaker, and Gopinaath Kannabiran. 2019. Queer(Ing) HCI: Moving Forward in Theory and Practice. In Extended Abstracts of the 2019 CHI Conference on Human Factors in Computing Systems (Glasgow, Scotland Uk) (CHI EA '19). Association for Computing Machinery, New York, NY, USA, 1-4. https://doi.org/10.1145/3290607.3311750 
[81] Iraklis Stamos, Evangelos Mitsakis, and Theodore Tsekeris. 2016. Harnessing the opportunities of austerity: a detailed mapping of the Greek transportation sector. Tema. Fournal of Land Use, Mobility and Environment 9, 3 (2016), 269-286.

[82] Arun Sundararajan. 2016. The sharing economy: The end of employment and the rise of crowd-based capitalism. Mit Press.

[83] Michael K. Svangren, Mikael B. Skov, and Jesper Kjeldskov. 2018. Passenger Trip Planning Using Ride-Sharing Services. In Proceedings of the 2018 CHI Conference on Human Factors in Computing Systems (Montreal QC, Canada) (CHI '18). Association for Computing Machinery, New York, NY, USA, 1-12. https://doi.org/10.1145/3173574.3174054

[84] Roger F Teal. 1987. Carpooling: who, how and why. Transportation Research Part A: General 21, 3 (1987), $203-214$.

[85] Adi Tedjasaputra and Eunice Sari. 2016. Sharing Economy in Smart City Transportation Services. In Proceedings of the SEACHI 2016 on Smart Cities for Better Living with HCI and UX (San Jose, CA, USA) (SEACHI 2016). Association for Computing Machinery, New York, NY, USA, $32-35$. https://doi.org/10.1145/2898365.2899800

[86] Niels Van Doorn. 2017. Platform labor: on the gendered and racialized exploitation of low-income service work in the 'on-demand'economy. Information, Communication \& Society 20, 6 (2017), 898-914.

[87] Vasillis Vlachokyriakos, Clara Crivellaro, Pete Wright, Evika Karamagioli, Eleni-Revekka Staiou, Dimitris Gouscos, Rowan Thorpe, Antonio Krüger, Johannes Schöning, Matt Jones, Shaun Lawson, and Patrick Olivier. 2017. HCI, Solidarity Movements and the Solidarity Economy. In Proceedings of the 2017 CHI Conference on Human Factors in Computing Systems (Denver, Colorado, USA) (CHI '17). ACM, New York, NY, USA, $3126-3137$. https://doi.org/10.1145/3025453.3025490

[88] Vasillis Vlachokyriakos, Clara Crivellaro, Pete Wright, and Patrick Olivier. 2018. Infrastructuring the Solidarity Economy: Unpacking Strategies and Tactics in Designing Social Innovation. In Proceedings of the 2018 CHI Conference on Human Factors in Computing Systems (Montreal QC, Canada) (CHI '18). Association for Computing Machinery, New York, NY, USA, 1-12. https://doi.org/10.1145/3173574.3174055 